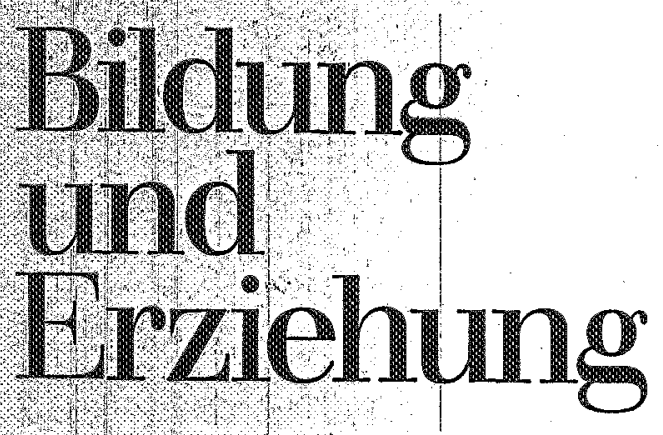

Sprachliche Kommunikation und schulerorientierter Unterricht
Spanhel

Praxisorientierte

Unterriehtsforschung

Lengert

Großere Sprechbetelligung der Schüler

Walcher

Verbale Instruktion

Bachmair

Ethnomethodologie

Loch

Curriculare Kompetenz

Forum:

Vaideanu

Problematik der modernen Welt

Dokumentation 


\section{Bildung und Erziehung}

Begründet von FraNz HiLKer $\dagger$.

Herausgeber

OsKaR ANWEILER, Ruhr-Universität, Bochum / OTTO EWERT, Johann-Gutenberg-Universität, Mainz / KarL Frex, Institut für Pädagogik der Naturwissenschaften an der Universität, Kiel / JoAchim KNoLl, Ruhr-Universität, Bochum / RuDOL F LENGERT, Universität Oldenburg / FrITz LOSER, Universität Osnabrück / WOLFGANG MrTTER, Deutsches Institut für Internationale Pädagogische Forschung, Frankfurt / Harm Paschen, Pädagogische Hochschule, Kiel / WILHELM RoEssLER, Ruhr-Universität, Bochum.

\section{Redaktion}

Dr. Manpred Heinemann, Markstraße 260, 4630 Bochum 1 (Telefon $0234 / 72334$ ).

Bildung und Erziehung erscheint zweimonatlich und kann direkt vom Verlag oder durch jede Buchhandlung bezogen werden. Preis des Einzelheftes DM 11,50, im Abonnement jährlich DM 58,-, für in Ausbildung stehende Lehrkräfte und Studierende im Abonnement Jahrlich DM 48,-, Vorzugspreis bei Abnahme von mindestens 10 Heften einer Nummer DM 9,20; jeweils einschließlich 6\% Mehrwertsteuer, zuzüglich Porto. Das Abonnement verlängert sich automatisch, wenn die Kündigung nicht zum 1. Dezember erfolgt ist. Zuschriften, die den Vertrieb und die Anzeigen betreffen, an den Verlag erbeten. Für der Redaktion unverlangt eingesandte Manuskripte und Besprechungsexemplare kann keine Haftung übernommen werden. Alle Rechte für sämtliche Beiträge vorbehalten.

Adressenänderungen: Bezieher, welche die Schriftenreihe direkt vom Verlag beziehen, bitten wir, die neue Anschrift zusammen mit der alten rechtzeitig mitzuteilen.

Anzeigen: Zur Zeit ist die Anzeigenpreisliste Nr. 7 vom 1. Jan. 1979 gültig. Verantwortlich für den Anzeigenteil: Hans-Jürgen PLAppert Anzeigenverwaltung Klett-Cotta Verlag: Gisela Lutz, Rotebühlstr. 77, 7000 Stuttgart 1 Telefon: 0711 / $6672-647$ od, 662

Verlag: Verlagsgemeinschaft Ernst KlettJ. G. Cotra'sche Buchhandlung Nachfolger GmbH., Postfach 809, 7000 Stuttgart 1.

Gesamtberstellung: W. Röck, Weinsberg ISSN 0006-2456

\section{Inhalt}

Beiträge:

Rudolf Lengert:

Sprachliche Kommunikation und schülerorientierter Unterricht

$\mathrm{Zu}$ diesem Heft $\ldots \ldots \ldots \ldots \ldots \ldots$

Dieter Spanbel:

Unterrichtsanalyse im Rahmen praxisorientierter Unterrichtsforschung .....

\section{Rudolf Lengert:}

Zur Rechtfertigung einer größeren

Sprechbeteiligung der Schüler im

Unterricht $\ldots \ldots \ldots \ldots \ldots \ldots \ldots \ldots$

Klaus Peter Walcher:

Verbale Instruktion im Rahmen eines kognitiven Modells ...............

\section{Ben Bachmair:}

Ethnomethodologie als handlungstheoretische Grundlage einer Didaktik der Kommunikation

Ein Diskussionsbeitrag

Werner Loch:

Curriculare Kompetenz und pädagogische Paradigmen ............. 241

Forum:

\section{George Vaideanu:}

Die Problematik der modernen Welt, das Curriculum und die ständige Weiterbildung; methodologische Betrachtungen 267

Dokumentation:

Zukunftsaufgaben der

Bildungspolitik $\ldots \ldots \ldots \ldots \ldots \ldots \ldots 274$

Berichte und Kurzmeldungen ...... 280

\section{Beilagenbinweis}

Diese Ausgabe enthält Beilagen der Edition Hirthammer, München und der Verlagsgemeinschaft Klett-Cotta, Stuttgart. Wir bitten unsere Leser um freundliche Beachtung. 


\title{
Ben Bachmair
}

\section{Ethnomethodologie als handlungstheoretische Grundlage einer Didaktik der Kommunikation}

\author{
Ein Diskussionsbeitrag
}

Summary: Our focus is the question as to how communication in school and instruction can be organized in an open-ended yet methodical fashion. Accordingly we proceed from the thesis that teaching methodology must be in accordance with the methodology of everyday activities so that school instruction can build on the structure of reflexive communication. We therefore adopt ethnomethodology as a theory of everyday activities in order to establish a repertoire of teaching methodologies. Taking an ethnomethodological perspective we analyze two examples of pupil aggression from the aspect of the reflexivity of accounts and develop suggestions for openended teaching methodologies.

Was ist Ethnomethodologie und wie kann man sie didaktisch auswerten? Dazu zwei Beispiele, die mit Hilfe der Ethnomethodologie analysiert und unterrichtsmethodisch umgesetzt werden sollen. Bei allen drei Beispielen geht es um Aggressionen, die im Schulalltag in dieser oder jener Form Probleme machen, die aber in der Schule zumeist nur mehr oder weniger zufällig und ohne explizite didaktische Theorie angegangen werden.

\section{Aggressionen am Rande des Unterrichts}

In einer 4. Klasse Grundschule, nach einer kurzen Pause', zwei Stunden Kunsterziehung stehen auf dem Stundenplan. Ein Kind, ein türkisches Mädchen, fehlt. Es ist nidht mehr aus der Pause in die Klasse zurückgekommen, weil es auf dem Schulhof verprügelt wurde. Der Lehrer sucht das Kind. Anschließend fragt er die Klasse, was denn los war; die Kinder berichten; der Lehrer bewertet die Prïgelei auf dem Schulhof. - Der Kunstunterricht beginnt. Nach einer Stunde ist Pause. Jetzt sagt ein kleiner, schmächtig aussehender Junge im Vorbeigehen zu dem vorher verprügelten Mädchen (das einen sehr kräftigen Eindruck macht): „Wenn du mir nochmal meine Jacke runterwirfst, mach ich dich tot ${ }^{\prime \prime}$.

\section{Auf dem Schulbof nach dem Unterricht}

Zwei Sieben- oder Adhtjährige liegen am Boden und prügeln sich. Eine Reihe von Kindern steht herum. Dann holt der eine einen Stock, der andere einen Stein und gehen wieder aufeinander los. Plötzlich fällt der Junge mit dem Stock hin und fängt unvermittelt $z u$ weinen und zu jammern an: "Au, au, mein Knie". Daraufhin suchen die umstehenden Kinder das Weite. Eine unbeteiligte Frau geht zu dem weinenden Jungen. Er läßt sich bereitwillig sein Knie anschauen, läßt sich trösten und streicheln; plötzlich beginnt er sehr viel zu erzählen: die anderen ärgern ihn dauernd, gehen immer auf ihn los; aber er gibt's ihnen schon zurück: „denen

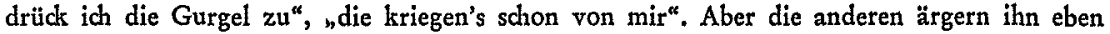
immer und hänseln ihn dauernd. Die Frau bestätigt den Jungen in seiner Darstellung, sagt $\mathrm{ihm}$, sie könne ihn verstehen, daß er sich da schlecht fühlt. Daraufhin wird er sehr traurig und sagt, daß es ihm gar keinen Spaß macht, in die Schule zu gehen: „Ne andere Schule wär’ 
vielleicht besser, ich mag gar nicht mehr hierher. Hierher gehe ich nicht mehr". Frage der zuhörenden Frau, ob er dies seiner Lehrerin schon einmal gesagt hätte. ${ }_{n}$ Ja, die sagt aber nur, ,geschieht dir schon recht, wenn du immer so loshaust" ".

\section{Ethnomethodologie als Programm}

Wie geht Ethnomethodologie an diese oder ähnliche Beispiele heran? Spontan habe ich als Erwachsener Mitleid mit den von ihren Lehrern allein gelassen und irgendwie hilflosen Kindern. Andere werden die geschilderten Kinder für aggressiv, verhaltensgestört, falsch erzogen usw. halten. Die Ethnomethodologie geht anders an diese Probleme heran, was auch der Terminus „Ethnomethodologie ${ }^{\text {ef }}$ herausstellt. Im Kern geht es der Ethnomethodologie um die Methoden fremden Handelns, die es angemessen zu verstehen und zu interpretieren gilt.

"Ethno-" bedeutet: auch in scheinbar vertrauten Situationen wie ein Forscher vorgehen, der eine fremde Volksgruppe erkundet, indem er ihre Sprache erlernt, um von ,innen' heraus fremdes Handeln zu verstehen.

"-method-" bedeutet: der außenstehende Beobachter unterstellt dem beobachteten Handeln eine erkennbare Methode, die die Handelnden auch selber darstellen und kommentieren.

Die Methode des Handelns ist der Gegenstand der Ethnomethodologie.

"-logie" bedeutet: Handeln im Alltag ist kompetentes Handeln, und die Tätigkeit des Wissenschaftlers, das Erklären, ist seinem Wesen nach konstitutiver Teil des Alltagshandelns. Alltagshandeln ist das Handeln des Ethnomethodologen; jeder Handelnde ist Ethnomethodologe.

Wichtig für das Verständnis der Ethnomethodologie ist die Analogie des Eindringens in fremde Lebenswelten ${ }^{1}$, die für Erkunden der Methode des Alltagshandelns steht. Eine fremde Gesellschaft wird beobachtet, um zu verstehen, wie man dort lebt. Das wird von der Absicht geleitet, aus dem Verständnis der fremden Lebenswelt etwas für die Interpretation der eigenen Lebenswelt $z u$ lernen. Beobachtung und Forschung stehen hier im Kontext von Erfahrung und Kommunikation (vgl. Arbeitsgruppe Bielefelder Soziologen 1976). Es geht nicht darum, (scheinbar) objektive, d. h. überhistorisch allgemeingültige Aussagen zu formulieren, sondern um die Verschränkung von subjektiven Lebenswelten in der Form von Kommunikation, die auf der Reflexivitär des alltäglichen Handelns aufbaut. Hierzu bringt die Analogie „als Fremder in einen unbekannten Volksstamm eindringen " ${ }^{2}$ die wichtige Einsicht, daß man als "Ethnologe" zuerst die Sprache der Eingeborenen lernen muß, um damit den Bezugsrahmen des fremden Handelns zu entdecken.

Für unsere Beispiele bedeutet diese Analogie des Ethnologen folgendes: Der Erwachsene, d. h. der Lehrer, der Erziehungswissenschaftler, der hospitierende Student geht mit der Einstellung an Kinder heran, etwas von den Kindern lernen zu wollen. Das heißt als Erwachsener schaut man den Kindern zu und wertet nicht, weil man zuerst die Regeln und Ausdrucksformen der Kinder, quasi ihre eigene Sprache, begreifen muß. 


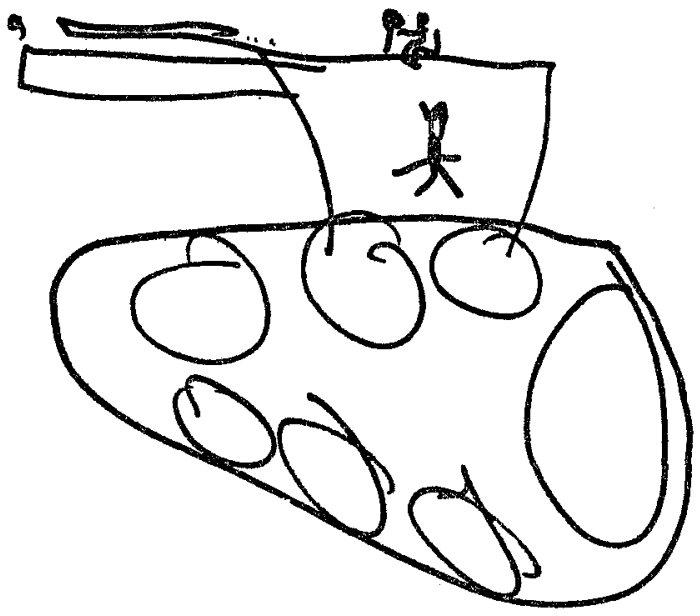

So ist dieser Panzer nicht die Kriegsmaschine des Erwachsenen. Die Zeichnung eines sechsjährigen Grundschülers stellt das Problem des Unterlegen-Seins aber Sich-Durchsetzen-Wollens dar: winzige $Z$ werge $(\rightarrow$ Symbol für Kinder) haben eine riesengroße Maschine, um sich damit in einer riesengroßen, ïberlegenen Welt durchzusetzen. Der "Panzer" entspricht dem „Knüppel aus dem Sack" des Märchens.

\section{Darstellen und Kommentieren als Strukturmomente des Handelns}

\subsection{Argumentation der Ethnomethodologie}

Diese Einstellung, sich gegenüber Kindern als Lernender zu verstehen, ist weder neu noch - zumindest theoretisch - umwälzend. Der Beitrag der Ethnomethodologie zur Aufklärung der Struktur des Handelns ist dagegen ein wichtiger Schritt, der zu einer Didaktik der Kommunikation Wesentliches mit beitragen könnte. Enthnomethodologie unterstellt dem von „außen“ beobachteten Handeln Methode. Die Kinder unserer Beispiele handeln methodisch. Zuschlagen, Drohen, Weinen, Reden usw. sind einmal Mitteilungen, zugleich aber auch Erklärungen, mit deren Hilfe die Kinder - ebenso wie alle anderen Gesellschaftsmitglieder - „ihr Verhalten und das anderer als Ereignis einer sozialen Ordnung (WIEDER/ZimmermanN 1976, S. 120) erkennbar machen. Die Mitteilung ist nicht nur Informationstransport, sondern erklärt zugleich auch den Handlungssinn und den Sinn einer Situation. (Dieses Erklären kann die Form des Darstellens und Kommentierens haben.) Der Sinn, den dieses aggressive Handeln in den skizzierten Situationen hat, entsteht mit diesen Erklärungen.

Zugleich ist diese Leistung des Erklärens eine Handlung in eben der Situation, die durch sie als Sinnerklärung konstituiert wird. Erklären „organisiert eine Welt, während sie zugleich in eben dieser Welt und auf eben diese Welt wirken" (W/EDER/ ZimMERMANN 1976, S. 121 f.). 
Diese Beziehung von Handeln, Erklären, Situation und Sinn wird im folgenden als Reflexivität bestimmt und anhand der Begriffe des Darstellens, des Kommentierens und der Indexikalität (Situationsbezug, Unausschöpfbarkeit einer Situation) analysiert. Eine entscheidende Annahme der Ethnomethodologie zur Struktur des Handelns ist also, daß dem Mitteilen und Erklären des eigenen Handelns die allgemeine Funlktion zukommt, Ordnung - d. h. eine Lebenswelt - herzustellen. Mitteilen und erklären wird somit zur ordnung- und realitätschaffenden menschlichen Handlung.

Menschen handeln also methodisch, indem sie den Sinn ihres Handelns mitteilen und erklären. WIEDER und ZiMMERMANN (1976, S. 107, S. 105) formulieren das als anthropologische Grundkonstante: „Die Ordnung, welche die Erdenmenschen in ihrem eigenen Tun entdecken, ist eine geschaffene Ordnung und wird durch ihre eigene Beschreibungsarbeit hergestellt." "Jede soziale Gruppe von Erdenmenschen ist dadurch charakterisiert, daß ihre Mitglieder fast ohne Unterlaß damit beschäftigt scheinen, sich selbst zu beschreiben und zu erklären."

Harold Garfinkex $\left(1967\right.$, S. 7) ${ }^{3}$ umschreibt diese Annahme folgendermaßen: Alltagshandeln ist so organisiert, daß es als "rational - erkennbar - und - berichtbar für - alle - möglichen - praktischen - Zwecke" dargestellt wird. Dieses Mitteilen und Erklären als eine wesentliche Methode menschlichen Handelns setzt zum einen die Darstellbarkeit (accountability, Garfinked/SAcks 1976, S. 132, S. 135, S. 167) des Sinns und der Methoden einer Handlung voraus.

Damit ist der uns geläufigere Begriff der Objektivierung, der Vergegenständlichung gemeint. Weil Regeln, Absichten, Handlungen symbolisch faßbar, also objektivierbar sind, kann man sie mitteilen.

Die zweite wesentliche Komponente des Mitteilens und Erklärens ist das Kommentieren (glossing practices, GarfrNKEL/SACKs 1976, S. 165). Mitteilen ist kein einmaliger Alkt, sondern steht in einem ständigen Prozeß des Kommentierens. Der darstellbare Sinn des eigenen Handelns wird mitgeteilt, steht damit quasi vergegenständlicht in einem situativen Handlungskontext, der zu einem Kommentar herausfordert. Man vergewissert sich laufend des eigenen und des fremden Handlungssinns im sich jeweils verändernden Handlungskontext, indem man die Situation kommentiert.

Zusammenfassend läßt sich herausstellen: Die Kinder handeln methodisch, indem sie sich mitteilen. Dabei stellen sie die Regeln ihres Handelns dar; die Darstellungen werden kommentiert.

\subsection{Folgerungen für die Schule}

Auf unser Beispiel des Jungens, der sich auf dem Schulhof prügelt, angewendet, bietet sich dann folgende Analyse bzw. Interpretation an. Zuprügeln, einen Stock holen, Weinen sind Mitteilungen und Erklärungen; sie sind einmal Darstellungen, deren Sinn es zu entschlüsseln gilt. Gleichzeitig kommentiert der Junge seine eigenen Darstellungen. So holt der Junge einen Stock, womit er (vermutlich) den Versuch, überlegen zu sein, gewinnen $z \mathfrak{u}$ wollen, darstellt. Das Weinen anschließend stellt (vermutlich) Angst und Hilflosigkeit dar. Das Weinen kommentiert aber auch die vorausgegangene Imponiersituation als ambivalent, daß eben auch Angst hinter der Drohgebärde stand. 
Als Erwachsener müßte man versuchen, diese Darstellungen zu entschlüsseln. Mit Sicherheit ist es falsch, Zuprügeln als Darstellung von Aggressivität zu interpretieren, auch wenn das in der gängigen Alltagspsychologie so gedeutet wird. Der Junge kommentiert durch sein Weinen sowohl sein Handeln wie die Kampfsituation als auch seinen eigenen Handlungssinn. Die eingreifende Frau interpretiert diesen „Kommentar" auch richtig als Darstellung von Angst, als Darstellung des Gefühls, abgelehnt, unterdrückt zu werden. Indem sie dem Jungen zuhört, stellt sie ihr Wohlwollen dar, weiteren Kommentaren zuzuhören. Der Junge wechselt die Darstellungsform und teilt dem Erwachsenen den Sinn seines aggressiven Handelns sprachlich-abstrakt mit, d. h. weitgehend in der "Sprache" des Erwachsenen.

Analysiert man das erste Beispiel (das Gastarbeiterkind wird verprïgelt, der schmächtige Junge droht später, sie "tot zu machen") unter den Gesichtspunkten des Darstellens und Kommentierens, so lassen sich Hinweise für eine unterrichtsmethodische Umsetzung des Darstellens und Kommentierens gewinnen. Daß das Gastarbeiterkind verprïgelt wurde, macht der Lehrer zu Beginn des Kunstunterrichts sozusagen zum Unterrichtsgegenstand. So wichtig das ist, es geht aber an der "Sprache" der Kinder vorbei; das Prügeln wird vom Standpunkt des Erwachsenen aus moralisch und dazu in der Form des gelenkten Unterrichtsgespräches thematisiert. Unterricht schließt auf diese Art und Weise nicht an den Darstellungen der Kinder an. Der "Kommentar" des Schülers „... dann madn' ich dich tot" zeigt das deutlich. Dieser Kommentar stellt etwas dar, was erst zu entschlüsseln wäre. Dazu müßte man weitere Darstellungen und Kommentare beobachten, um so das Problem der Kinder und ihre Absichten zu verstehen. Es stellt sich also die Aufgabe, ein Unterrichtsarrangement zu finden, das einmal Beobachtungsphasen einschließt, um das Defizit des Lehrers zu kompensieren. Dieses Arrangement müßte darüber hinaus die Kinder zum eigenen Darstellen und Kommentieren anregen. Diese eigenen Kommentare der Kinder liegen in dieser Situation so weh das einem Erwachsenen tun mag - beim „... dann mach' ich dich tot“. Da der Situationskontext nur unzureichend bekannt ist, und dieser Kontext ist konstitutiv für Darstellen und Kommentieren, läßt sich bestenfalls die unterrichts-methodische Richtung zeigen, wie die Kinder die Darstellung des Prügelns in ihrer Sprache kommentieren könnten. Statt des ursprünglichen Themas des Kunstunterrichtes „Segelschiffe", würde es sich möglicherweise empfehlen - spontan - die Schule und das Klassenzimmer als Bildthema zu stellen. Im Rahmen dieses Themas hätten die Kinder zumindest die Gelegenheit, das Geschehen auf dem Schulhof zu kommentieren, vermutlich verdeckt, nachdenkend, ohne das Geschehen sprachlich oder bildlich darzustellen. In der Klasse vorsorglich gesammelte Aggressionssymbole (z. B. Kinderzeichnung mit dem Panzer, entsprechendes Spielzeug usw.) wären ebenfalls in der Lage, die angesprochene Unterrichtsfunktion zu übernehmen. Allgemein bieten sich Bild-, Foto-, Zeichenwände als Anregungen für Schüler an, laufend ihr Schulleben zu kommentieren. Zwar wird man als Erwachsener von diesen Kommentaren wenig erfahren, weil sie am Rande und verdeckt laufen. Wichtiger ist jedoch, daß die Schüler die Chance dazu bekommen - auch und gerade wenn der Lehrer den Prozeß nicht kontrollierend führt, sondern nur Möglichkeiten arrangiert.

Die Fotowand ist in diesem Zusammenhang als Beispiel eines organisierten Unterrichtsarrangements $z \mathfrak{u}$ verstehen, das der Struktur des Handelns entspricht. Fotos, Bilder

BuE 32 (1979) 3 
usw. ermöglichen das Kommentieren von Handlungsintentionen, Handlungen und Situationen, wobei Kommentieren als notwendige Grundlage komplexer Formen der Auseinandersetzung anzusehen ist.

Der zweite Aspekt methodischen Handelns, das Darstellen der Regeln des Handelns durch das Handeln als "Medium" wäre ebenfalls in Unterrichtsarrangements umzusetzen. Diese Arrangements müßten auf dem Prinzip des Darstellens, der symbolischen bzw. handelnden Entäußerung und Objektivierung von Handlungsmustern aufbauen. Das traditionelle Methodenrepertoire der Didaktik reicht dazu nur schwer aus, denn es ist in der Regel von der Struktur des zu lehrenden Sachverhaltes hergeleitet, womit diese Methoden dem Handlungskontext der Kinder eher fremd sind, also auch nicht in das „Sprach-“ bzw. Darstellungsrepertoire der Kinder passen. Sozialpädagogische Methoden mit ihrer Klientenzentrierung (z. B. Aktives Zuhören, Rollenspiele, offene musikalische Darstellungsformen, offenes Zeichnen usw.) entsprechen eher dem skizzierten Konzept methodischen Handelns. Audiovisuelle Medien, die auch in außerpädagogischem Verständnis Situationen und Intentionen darstellen und festhalten, haben ebenfalls deutliche Affinität zu didaktischen Arrangements, die kindliche Darstellungen von Handlungsmustern begünstigen 4 .

\section{Reflexivität als Strulkturelement des Handelns}

Die Aussage des schmächtigen Jungen aus unserem Beispiel vom Kunstunterricht „Wenn du mir nochmal meine Jacke runterwirfst, mach' ich dich tot" läßt sich als Kommentar zu den Ereignissen am Rande des Unterrichtes deuten. Uber dieses Beispiel hinaus wurde Kommentieren als Form der eigenständigen Auseinandersetzung beschrieben, die durch organisierte Arrangements unterstïtzt werden sollten. Das Beispiel zeigt aber deutlich, daß die kommentierende Form der Auseinandersetzung die Verstrickung in ein komplexes Geschehen nicht berührt. Ziel didaktischer Maßnahmen wäre es doch, daß die Kinder - und natürlich auch die Lehrer wie die beobachtenden Wissenschaftler oder Studenten - sich dieser ihrer Verstrickung bewußt werden, um sich so daraus zu befreien. Der schmächtige Junge sollte sein Problem bzw. sein Handlungsmuster verstehen, das ihn zum Kommentar "Totmachen" veranlaßt. Ebenso ist der Lehrer aufgerufen, sich seinen Beitrag zu diesen Kommentaren vor Augen zu führen, denn er hat durch sein Festhalten an einem für die Kinder in dieser Situation unwesentlichen Lernziel den Schülern die Chance der Auseinandersetzung mit einem für sie wichtigen und aktuellen Problem genommen. Glaser und Strauss (1974) bezeichnen diesen Problempunkt als den Grad der Bereußtheit, mit dem man eine Situation und die hier zugrundeliegenden Handlungsmuster versteht. In unseren drei Beispielen ist der Grad der Bewußtheit der Kinder minimal; sie bleiben in ihre Situation und ihre Handlungsmuster verstrickt. Die Beispiele zeigen jedoch auch "Stellen", wo die Situationen brïchig werden. Das mit dem Stock drohende Kind fällt hin, es weint, ein Erwachsener hört zu, das Kind beginnt von sich und seinen Problemen zu erzählen. Hier bietet sich dem Kind die Chance, seine Intentionen sich bewußter zu machen. Der Umstand, daß ihm nur einmal jemand und eigentlich ganz zufällig zuhört, wird diese Chance jedoch wieder verschütten. Es müßte ein didaktisches Arrangement gefunden werden, Situa- 
tionen organisiert methodisch zu reflektieren und aufzubrechen ${ }^{5}$. Solche Arrangements setzen bei den Darstellungen der Kinder (Prügeln, Weinen, Reden, Zeichnungen usw.) an.

Darstellungen sind jedoch unlösbar mit einer Situation verbunden. Sie stehen in einem situativen Kontext und verändern diesen Kontext. Darstellungen erscheinen immer in einem speziellen Kontext, der die entäußerte Darstellung integriert und somit zu etwas Neuem macht, was zugleich zur Herausforderung für die Kinder werden kann. Ethnomethodologie verwendet hier den Schlïsselbegriff der Indexikalität (z. B. Garfinkel 1976, S. 143 ff., Cicourel 1975, S. 37 ff., Weingarten/SACK 1976, S. 14 ff.). Das Phänomen der Indexikalität, des unauflöslichen Situationsbezuges wird auch sprachlich abgebildet. Als Beispiel eine mögliche Aussage: „Aber so geht das nicht. So kann man das nicht machen." Indexikalische Ausdrücke (hier kursiv) sind nur aus der Situation heraus eindeutig verstehbar. Das "Aber" verweist auf etwas Vorangegangenes, von dem ein Sprecher einen in der Sprechsituation eindeutigen Sachverhalt "das" und seine Regeln "so" und die Verursacher dieses Sachverhaltes "man" kritisierend abhebt. Diese Aussage, die nur im Rahmen der uns nicht bekannten Situation eindeutig würde, fordert $\mathrm{zu}$ einer Stellungnahme heraus. Hier läßt sich eine Art von Spannung zwischen Situation und der Darstellung des Handlungsmusters feststellen. Für den Wissenschaftler führt diese Spannung z. B. zum Versuch, eine Aussage möglichst eindeutig zu formulieren und "Aber" "das" „so" "man" durch eindeutige Aussagen zu ersetzen (vgl. Garfinkel 1973b, S. 201 ff.). Die Spannung zwischen dargestelltem Handlungsmuster und Situation fordert Kinder, im Gegensatz zum Wissenschaftler, nicht zum Eindeutig-Machen heraus. In unserem Beispiel, zwei Jungen prïgeln sich, führt die Situation bzw. die Veränderung der Situation zu einem Wechsel der Darstellungsform (Prügeln - Weinen - Reden) und zu neuen Kommentaren.

Die Spannung $z$ wischen Indexikalität und Darstellung eines Handlungsmusters läßt sich als Reflexivität interpretieren (MeHAN/Wood 1976, S. 29 ff., CICOUREL 1975, S. 35 f., S. 115 f.). Reflexivität ist ein konstitutives Strukturelement alltäglichen Handelns, das Resultat der unaufheblichen Spannung zwischen Handlungsmuster und der situativen Erscheinungsform ist. Diese Spannung, diese Reflexivität, ist als Brechung von Handeln in Situationen zu verstehen, die das Bemühen auslöst, sich des Sinns eigenen Handelns zu vergewissern. Kommentieren ist die erste wesentliche Möglichkeit, den Sinn eigenen Handelns in der jeweiligen Situation zu reflektieren.

Für die Didaktik stellt sich die Frage, wie Situationen zu arrangieren sind, um Reflexivität organisiert methodisch mit dem Ziel auszuwerten, Kommunikation bewußter zu machen. Hierzu werden zwei Strategien vorgestellt.

Bevor auf strategische Möglichkeiten eingegangen wird, geht es um den entscheidenden theoretischen Vorzug der Ethnomethodologie als Handlungstheorie, nämlich eine genuin offene Didalstik der Kommunikation näher bestimmen zu können. Zumindest in der theoretischen Diskussion besteht Übereinstimmung dariber, daß die Thematisierung von Kommunikation in der Schule offen zu organisieren sei. Damit ist im wesentlichen gemeint, der Lehrer habe sich aus der Rolle des Entscheidungsträgers und Meinungsmachers herauszunehmen, um dem Schüler eigenständige Erfahrungsmöglichkeiten zu erschließen bzw. anzubieten. So gesehen, steht Offenheit gegen Verschulung. Zwischen organisiertem, institutionalisiertem Lernen und offener Kommu- 
nikation klafft demnach ein. Widerspruch, der entweder durch ein Minimum an Organisation und Planung oder durch die Delegation der Planung an die Schüler zu lösen ist. Theoretisch stellt sich jedoch die Aufgabe anders. Die widersprüchliche Beziehung zwischen Organisation und Planung einerseits und Kommunikation andererseits besteht nur oberflächlich. Handlungstheoretisch gesehen ist Offenheit am Moment der Reflexivität festzumachen. Reflexivität als Spannung zwischen Situation und Darstellung ermöglicht und exfordert offene Methoden. Sowohl situative Arrangements (vom Rollenspiel bis zur Aktiven Medienverwendung) als auch die „Brechung“ von Darstellungen mittels objektivierender Dokumente (z. B. Tonbandprotokolle, Sofortbilder, schriftliche Protokolle) unterstützen das allgemeingültige reflexive Moment des Handelns.

\section{Organisiert methodische Brechung}

Didaktische Aufgabe ist es demnach, Reflexivität als Strukturelement des Handelns organisiert methodisch zu unterstïtzen. Das Ziel liegt darin, Bewußtheit von Kommunikation in der Schule und im Unterricht zu begünstigen. Dieses Ziel gilt es im Auge zu behalten, um entsprechende Methoden nicht zu einer Kommunikationstechnologie verkommen $\mathrm{zu}$ lassen.

Es sind zwei Typen von Strategien denkbar, die "methodische Brechungen" organisieren. Den einen Verfahrenstyp hat Cicourel (1975, S. 156-166) beschrieben. Es handelt sich dabei um fortlaufendes Verfahren der Konfrontation audiovisueller Situationsdokumente mit Kommentaren. Beim zweiten Verfahrenstyp geht es um die Konfrontation von Situationsdokumenten mit Taxonomien, die Handlungsmuster überschaubar zusammenfassen.

\subsection{Die unbegrenzte Konfrontation von Situationsdokumenten und Kommentaren}

CrCourel läßt dabei verschiedene Dokumente zu einer Situation erstellen, z. B. die Videoaufnahme einer Unterrichtsstunde, den Bericht eines Lehrers, Berichte der Schüler, Pläne und Erwartungen zur Unterrichtsstunde. Diese Berichte werden den Beteiligten wiederum mit der Bitte zur Verfügung gestellt, ihre Meinung, Einschätzung, Bewertung zu formulieren: Die verschiedenen Dokumente wie die verschiedenen Kommentare zu den Dokumenten stellen jeweils unterschiedliche Aspekte der Situation heraus. "Jeder Bericht bezieht sich auf erwähnte Einzelheiten und hängt von den sozialen Beschränkungen der aktuellen Unterrichtsstunde ab. Unsere selektive Informationsspeicherung, die Veränderung der Erinnerung an den Situationszusammenhang oder die selelktive bzw. sich verändernde Wiederherstellung des Sinnzusammenhangs, die auf Tonband oder Video aufgezeichnet wird, bietet eine wichtige Basis für unbegrenzte Berichte von ,derselben' Szene ${ }^{c t}$ (Crcourel 1975, S. 166). Das Phänomen der Indexikalität, daß Situationen nie ausschöpfbar, d. h. nie eindeutig beschreibbar sind, nötigt zu immer neuen Varianten der Beschreibung in Form von Kommentaren, die ihrerseits zu Dolkumenten, somit zu Beschreibungsvarianten werden. In diesem unendlichen Prozeß liegt die Chance der Auseinandersetzung mit Kommunikation. Die- 
ses unbegrenzte Auseinandersetzungsverfahren (Crcourel 1975 nennt es Triangulation) „versucht den praktischen Sinn und die inhärente Reflexivität alltäglicher Erzählungen sichtbar zu machen" (CICOUREL 1975, S. 158).

In einer Schule schiene mir solch ein unbegrenztes Auseinandersetzungsverfahren am einfachsten über Fotografie zu realisieren. Die mittlerweile kinderleichte Technik (z. B. Sofortbilder mit fix-focus-Kameras), der einfache Zugriff (in den meisten Familien ist irgendein Fotoapparat auch für Kinder im Prinzip verfügbar) und der Vorzug, Bilder problemlos in der Schule zu veröffentlichen (Schaukästen, Fototafeln), sprechen für die Fotografie. Hinzu kommt, daß der notwendigerweise distanzierende Blick durch den Sucher Situation neu erleben läßt. Das heißt, das Phänomen der Indexikalität regt Kinder dazu an, sich der Situation interpretierend zu vergewissern (Reflexivität). Damit erhöht sich für Kinder die Chance, in den Prozeß des Kommentierens und Dokumentierens einzusteigen.

In eng begrenzten Fällen läßt sich dieses Auseinandersetzungsverfahren direkt in den Unterricht integrieren (vgl. unser Beispiel aus dem Kunstunterricht, Punkt 2.2). Das ist aber in der Regel nur für solchen Unterricht möglich, in dem "Dolkumente" erstellt werden, z. B. in den Fächern Deutsch, Kunst, Musik, Sport, Werken.

Der $z$ wreite und wesentlichere didaktische Ort liegt am Rande des Unterrichts, was zeitlich wie räumlich gemeint ist: in der Pause, in Zwischenstunden, auf dem Flur, auf dem Schulhof, im Treppenhaus usw. Diese Zeiten bzw. Räume bieten sich für ein offenes Auseinandersetzungsverfahren - gerade für Fotografie und Collage - an, weil diese Bereiche nicht durch feste und geschlossene Unterrichtsstrukturen bzw. Curricula belegt, dafür aber verhältnismäßig offen für eigenständige Aktivitäten der Schüler sind.

\subsection{Konfrontation von Situationsdokumentation mit Taxonomien}

Mit dieser Strategie versuche ich das Moment der Reflexivität über die Spannung zwischen audiovisuell, also realitätsnah dokumentierten Situationen und dem Handlungsmuster, das in der Situation dargestellt wird, zu unterstïtzen. Das Handlungsmuster wird in die abstrakte und einfache Form einer Taxonomie gebracht. Eine Taxonomie entspricht im wesentlichen der allseits geläufigen Form der Gliederung (z. B. ein Inhaltsverzeichnis), die einen komplexen Sachverhalt überschaubar geordnet auflistet. Eine bekannte, formell in die Didaktik eingeführte Taxonomie ist $\mathrm{z}$. B. die Lernzieltaxonomie von Broom, die aus folgenden Hauptkategorien besteht: Kenntnis, Verständnis, Anwendung, Analyse, Synthese, Bewertung. Diese Taxonomie bildet ganz abstrakt und formal Unterricht unter dem Aspekt des Lernziels ab. Das Heimannsche Schema zur Unterrichtsplanung (Intention, Inhalt, Methode, Medien, anthropologischpsychologische und situativ-soziale-kulturelle Bedingungen) stellt ebenfalls ein einfaches Unterrichtsmuster in Form einer Taxonomie dar.

Im Prinzip läßt sich jeder Aspekt von Unterricht auf die Form der Taxonomie bringen. Gewinnen lassen sich Taxonomien über eine theoretische Analyse; theoretische Aussagen werden dann auf ein einfaches Gliederungsschema reduziert. Solch eine Taxonomie hat dann die Funktion, theoretische Analyse mitteilbar und handhabbar zu machen. Kommunikationsfunktion bekommt die Taxonomie jedoch nur in Ver- 
bindung mit einem Situationsdokument, das seinerseits durch die Konfrontation mit der Taxonomie - als schlichtester Form einer Theorie - fragwürdig wird. Der Gegensatz konkret-situativ, abstrakt-allgemein unterstützt hier Reflexivität, indem ein Prozeß der Auswertung des Dokumentes und der Veränderung der taxonomisch gefaßten Theorie gleichzeitig beginnt. Das Problem dieser Strategie liegt im Aufwand, Taxonomien begründet so zu formulieren, daß sie auch im praktischen Kommunikationskontext verständlich sind. Dieser Aufwand ist zumindest überall dort notwendig, wo isolierte Dokumentationsverfahren mit dem Anspruch, Unterricht objektiv, valide und reliabel zu repräsentieren, entstanden sind. Im Rahmen der Lehrerausbildung gibt es mittlerweile ein solches System der Unterrichtsdokumentation, bei dem mit immer mehr Audio- und Viedeotechnik möglichst viele, am liebsten alle Aspekte des Unterrichts mit dem Anspruch auf Objektivität, Validität und Reliabilität abgebildet werden. Dem liegt die Annahme zugrunde, eine Unterrichtssituation sei letztlich unabhängig vom verwendeten Dokumentationsverfahren, dem zugrundeliegenden Interpretationsschema der Produzenten sowie dem Interpretationsschema der Verwender tepräsentativ darstellbar. Für diese Art von Unterrichtsdokumentation ist es geboten, die jeweiligen Interpretationsrahmen über Taxonomien zu thematisieren, um so in einen Auseinandersetzungsprozeß einzusteigen. Taxonomien haben hier die Kommunikationsfunktion, Kommentare zu Dokumenten wie didaktischen Theorien zu evozieren.

\section{Schlußbemerkung zur Problematik professioneller Wissenschaft}

Die Tätigkeit des professionellen Wissenschaftlers hat - im Sinne der Ethnomethodologie - nur scheinbar einen wahrheitsfördernden Sonderstatus. Das wesentliche der Tätigkeit des Wissenschaftlers entspricht der allgemeinen Struktur menschlichen Handelns, die immer auch reflexiv ist. Reflexivität des Handelns trifft für Lehren, wie für Lernen, wie für Forschen $\mathrm{zu}$, indem alle Beteiligten sich und andere beobachten, beschreiben, interpretieren. Die Komplexität des aus dem Alltagszusammenhang abgetrennten Lehrens und Lernens hat für die dabei notwendige Beobachtungs-, Beschreibungs- und Interpretationsarbeit einen eigenen Beruf entstehen lassen. Die gesellschaftliche Funktion der Abtrennung dieser reflexiven Aufgaben aus dem Unterrichtsalltag wird dann gern mit der daraus resultierenden Distanz gerechtfertigt, wobei Distanz wiederum als wahrheitsfördernd angesehen wird.

Abtrennung reflexiven Handelns von der Schule und Distanz zur Schule führen zur Entwicklung eines eigenständigen Regelwerkes, insbesondere zu einem eigenständigen Methodenapparat, der im Vergleich zu den Methoden alltäglichen Beschreibens und Erklärens ungeheuer komplex ist und damit die Vermutung unterstützt, objektive, valide und reliable Aussagen produzieren zu können.

In der Pädagogik ist wieder ein Punkt erreicht, an dem der scheinbare Vorteil isolierter Wissenschaft (Distanz, eigenständiger Methodenapparat) als Fetisch erfahren wird. Hier bietet sich die Chance, die gemeinsame Grundlage theoretischen wie praktischen Handelns und Erkennens zu suchen. In dieser Argumentation steckt aber ein höchst problematischer Punkt. Im Bereich der Pädagogik wird immer mehr professionellen Wissenschaftlern durch das Zusammenbrechen von Reform- und Innovationsidealen 
die eigene isolierte wissenschaftliche Arbeit fragwürdig. Es liegt der Versuch nahe, den Alltag als feste Basis für Wissenschaft zu benutzen. Wenn Wissenschafr - ob Unterrichtstechnologie, Curriculum, empirische Unterrichtsforschung - schon nicht tragfähig ist, sondern mehr als Folge wechselnder Moden erlebt wird, dann könnte der Alltag des Unterrichts und der Erziehung eine neue feste Basis, quasi einen Archimedischen Punkt, abgeben. Abstrakter gesagt, wenn Wissenschaftler ihre Arbeit als Fetisch, als entfremdet erleben und nicht als notwendigen Beitrag, Schule und Erziehungsinstitutionen an die Bedürfnisse der Kinder anzupassen, sondern als funktionierendes Durchsetzungsinstrument für Bildungsverwaltung, Verbände usw., dann läßt man sich auf die Praxis, den Alltag als etwas ein, in dem noch integriertes, überschaubares Handeln möglich erscheint, um dann mit dieser Beschreibung und ihrer impliziten Idealisierung ein neues wissenschaftliches Selbstverständnis zu gewinnen.

Damit wäre ein neuer Fetisch, die alltägliche Praxis, entstanden, was mehr Fluchtreaktion denn Aufklärung und Anleitung der Praxis bedeutet. Diese Gefahr steckt jedoch auch implizit im idealistischen Handlungsbegriff der Ethnomethodologie. Trotz dieser idealistischen Ausrichtung lassen sich aus der Ethnomethodologie unterrichtsmethodische Anregungen entwickeln, die jedoch allein kein theoretisches Fundament einer Didaktik der Kommunikation abgeben.

\section{Anmerkungen}

1. Vgl. hierzu den Ansatz von A. Scrürz (u. a. 1974), in der aktuellen Auswertung z. B. bei T. Heinze / Klusemann (1978).

2. Diese Analogie hat C. CAstaneda (u. a. 1971, 1973) in einem Roman verwendet, um sein Interesse, das mit dem der Ethnomethodologie korrespondiert, darzustellen.

3. Vgl. Wetngarten / SACK 1976, S.17.

4. Die im Rahmen des Reutlinger CIEL-Projelts (GIEL u. a. 1974/1975) skizzierte Theorie der Unterrichtsmethoden und -medien (Mehrperspektivische Rekonstruktion) ließe sich als Grundlage einer Ausdifferenzierung des Methodenrepertoires verwenden.

5. In der Ethnomethodologie führt diese Überlegung zum sog. Krisenexperiment, dessen Ziel es ist, den Alltag und dessen Bewältigung als brüchig erscheinen zu lassen. Vgl. GarrinkBL 1967, S. 42 ff., 1973a, S. 281; MEHAN / WoOd 1976, S. 51 f.

\section{Literatur}

Arbertrsgruppe Bielefelder Sozrologen (Hrsg.): Kommunikative Sozialforschung. München 1976.

Castaneda, Carios: Eine andere Wirklichkeit, Neue Gespräche mit Don Juan. Frankfurt 1971.

Castaned, Carlos: Die Lehren des Don Juan, Ein Yaqui-Weg des Wissens. Frankfurt 1973.

CrCourel, AAron V.: Sprache in der sozialen Interaktion. München 1975.

GarminKel, Harold: Studies in Ethnomethodology. Englewood Cliffs 1967.

GarRinKEL, Harold: Studien über die Routinegrundlagen von Alltagshandein. In: STEINERT, (Hrsg.): Symbolische Interaktion. Stuttgart 1973, S. 280-293 (zit. als Garninkel 1973a). Garfinkel, Harold: Das Alltagswissen über soziale Strukturen und innerhalb sozialer 
Strukturen. In: Arbeitsgruppe Bielefelder Soziologen: Alltagswissen. Interaktion und gesellschaftliche Wirklichkeit, Band 1. Reinbek 1973, S. 189-214 (zit. als Garrinket 1973b).

GarpinKex, Harold / Sacks, Harvey: Uber formale Strukturen praktischer Handlungen. In: Weingarten u. a. 1976, S. 130-176.

Giel, Kraus / Hiller, Gotrhilf G. / KRämer, Hermann: Stücke zu einem mehrperspektivischen Unterricht, Aufsätze zur Konzeption. Bd 1 und 2. Stuttgart 1974 und 1975.

Glaser, B. G. / Strauss, ANselm: Interaktion mit Sterbenden. Göttingen 1974.

Heinze, Tromas / Krusemann, H.-W. (Hrsg.): Lebensweltanalyse von Fernstudenten, Theoretische und methodenpraktische Oberlegungen zur Interpretation narrativer Interviews. Werkstattbericht Fernuniversität Hagen 1978.

MrHan, Hugh / Wood, Houston: Fünf Merkmale der Realität. In: Weingarten u. a. 1976, S. 29-63.

SchütZ, Alpred: Der sinnhafte Aufbau der sozialen Welt; Frankfurt 1974

Wengarten, Elmar / Sack, Fritz: Ethnomethodologie. Die methodische Konstruktion der Realität. In: WeINGARTEN u. a. 1976, S. 7-26.

Weingarten, Elmar / SAck, Fritz / Schenkein, Jim (Hrsg.): Ethnomethodologie. Beiträge zu einer Soziologie des Alltagshandelns. Frankfurt 1976.

Wieder, LaWrence / Zimmerman, Don H.: Regeln im Erklärungsprozeß. Wissenschaftliche und ethnowissenschaftliche Soziologie. In: WEINGArTEN u. a. 1976, S. 105-129. 